INPLASY

PROTOCOL

To cite: Huang et al.

Effectiveness and Safety of

Yufeng Ningxin for the

treatment of Essential

Hypertension: A protocol for a

systematic review and meta-

analysis. Inplasy protocol

202110059. doi:

10.37766/inplasy2021.1.0059

Received: 16 January 2021

Published: 16 January 2021

Corresponding author:

Changgeng Fu

fucgbs@163.com

Author Affiliation:

Xiyuan Hospital of China

Academy of Chinese Medical

Sciences, Beijing 100091, China

Support: National Natural

Science Found.

Review Stage at time of this submission: Preliminary

searches.

Conflicts of interest:

None.

\section{Effectiveness and Safety of Yufeng Ningxin for the treatment of Essential Hypertension: A protocol for a systematic review and meta-analysis}

Huang, MY1; Long, LZ2; Deng, M3; Yu, ZK4; Qu, H5; Tan, L6; Peng, $Y X^{7}$. Fu, CG8.

Review question / Objective: Yufeng Ningxin (YFNX) is a commonly used Chinese patent medicine in treating essential hypertension. The objective of this protocol is to evaluate the effectiveness and safety of YFNX for the treatment of essential hypertension.

Information sources: We plan to conduct a systematic search of studies through the following databases: PubMed, EMBASE, Cochrane Library, Chinese National Knowledge Infrastructure (CNKI), Wanfang Database, Chinese Biomedical Literature Database (CBM) and Chinese Scientific Journal Database (VIP) from the start date of the databases to January 1, 2021. The retrieval terms include YFNX, hypertension and RCTs. We will also search grey documents and clinical trial registers including the Chinese Clinical Trial Register (ChiCTR), WHO International Clinical Trials Registry Platform (ICTRP) and the Clinical Trials, to supplement the electronic databases.

INPLASY registration number: This protocol was registered with the International Platform of Registered Systematic Review and Meta-Analysis Protocols (INPLASY) on 16 January 2021 and was last updated on 16 January 2021 (registration number INPLASY202110059).

\section{INTRODUCTION}

Review question / Objective: Yufeng Ningxin (YFNX) is a commonly used Chinese patent medicine in treating essential hypertension. The objective of this protocol is to evaluate the effectiveness and safety of YFNX for the treatment of essential hypertension.

Condition being studied: Essential hypertension is the leading risk factor for deadly cardio-cerebrovascular diseases such as strokes, heart failure, myocardial 
infarction, atrial fibrillation, etc. As a result, hypertension is a major cause of death in the world, inducing 10.4 million deaths every year. Lowering blood pressure (BP) can significantly reduce the premature morbidity and mortality of cardiocerebrovascular complications. Despite the adoption of conventional treatment measures such as calcium channel blocker, angiotensin converting enzyme inhibitor, angiotensin receptor blocker, $\beta$-blockers, diuretics, and so on, the prevalence of hypertension continues to increase, leading to an increasing trend in the incidence and mortality of cardio-cerebrovascular diseases worldwide. The number of patients with hypertension will increase by $15-20 \%$ and will approach 1.5 billion by 2025. Therefore, seeking new complementary and alternative therapies is of great significance for the prevention and treatment of hypertension. YFNX, extracted and processed from the Pueraria lobata, is a commonly used Chinese patent medicine for the treatment of hypertension. It has the function of resolving tetany and relieving pain, enhancing brain and coronary blood flow as well as lowering BP. However, there is still no strong evidence showing the effectiveness and safety of YFNX in treating hypertension.

\section{METHODS}

Participant or population: Patients with essential hypertension.

Intervention: The intervention group took YFNX or YFNX combined with conventional therapy. The various dosage forms of YFNX will include dripping pills, tablets, capsules, granules, etc.

Comparator: The control group accepted placebo and/or conventional therapy such as calcium channel blocker, angiotensin converting enzyme inhibitor, angiotensin receptor blocker, $\beta$-blockers, diuretics, etc.

Study designs to be included: All randomized controlled trials (RCTs) of YFNX in the treatment of essential hypertension will be included regardless of language and publication types.
Eligibility criteria: The participants included must have a diagnosis of essential hypertension according to the criteria published in '2020 International Society of Hypertension global hypertension practice guidelines', which is defined as systolic $B P(S B P) \geq 140 \mathrm{mmHg}$ and/or diastolic $B P(D B P) \geq 90 \mathrm{mmHg}$. The patient's age, gender, ethnicity and region will not be considered.

Information sources: We plan to conduct a systematic search of studies through the following databases: PubMed, EMBASE, Cochrane Library, Chinese National Knowledge Infrastructure (CNKI), Wanfang Database, Chinese Biomedical Literature Database (CBM) and Chinese Scientific Journal Database (VIP) from the start date of the databases to January 1, 2021. The retrieval terms include YFNX, hypertension and RCTs. We will also search grey documents and clinical trial registers including the Chinese Clinical Trial Register (ChiCTR), WHO International Clinical Trials Registry Platform (ICTRP) and the Clinical Trials, to supplement the electronic databases.

Main outcome(s): The reduction of SBP and DBP.

Quality assessment / Risk of bias analysis: The quality of RCTs will be assessed based on the version 2 of the risk-of-bias tool (RoB 2) for randomized trials in the Cochrane Handbook by two independent authors. The main items include randomization bias (random sequence allocation, allocation concealment), intervention bias, measurement bias, missing outcome data bias and reporting bias.

Strategy of data synthesis: The RevMan $\mathbf{5 . 3}$ software (Cochrane Collaboration, Copenhagen, Denmark) will be applied for data synthesis.

Subgroup analysis: If the included studies have obvious heterogeneity and there are more than 10 trials, the subgroup analysis will be conducted on the basis of the age, 
sex, the severity of hypertension and the dosage of YFNX.

Sensibility analysis: In order to ensure the accuracy of research conclusions, We will conduct a sensitivity analysis by eliminating each study individually or transforming the category of the effect model.

Country(ies) involved: China.

Keywords: Yufeng Ningxin, essential hypertension, protocol, systematic review, meta-analysis.

Contributions of each author:

Author 1 - Mingyan Huang.

Author 2 - Linzi Long.

Author 3 - Mi Deng.

Author 4 - Zikai Yu.

Author 5 - Hua Qu.

Author 6 - Ling Tan.

Author 7 - Yuxuan Peng.

Author 8 - Changgeng Fu. 